\section{Mental health survey of the adult population in Iran}

\author{
A. A. NOORBALA, S. A. BAGHERI YAZDI, M. T. YASAMY and K. MOHAMMAD
}

According to the World Health Organization, the next two decades may witness worldwide changes in the pattern of epidemiology of diseases. Non-communicable diseases such as mental disorders may replace infectious and communicable diseases as the leading factor in disability and premature death (Murray \& Lopez, 1996). A review of epidemiological studies of mental disorders in different countries shows that grossly different prevalence rates have been obtained as a result of differences in the tools employed, sampling methods, interview techniques and diagnostic classifications. A review of studies on the prevalence of mental disorders in Iran (Noorbala et al, 1998) indicates differences in prevalence rates throughout the country. Efficient planning for provision of mental health care in Iran requires basic demographic data and a survey of the country's health status. Our survey was intended to determine the population's mental health status, as well as providing the country's planning authorities with estimates of the dimensions of mental health problems.

\section{METHOD}

The population sample of this survey consisted of urban and rural dwellers in the age group 15 years and above. The country's population, according to statistics provided by the health system, was 63042188 in 1999 , of whom $64.2 \%$ lived in urban areas and $35.8 \%$ in rural areas. The total number of households was 12685113 .

\section{Sample}

Cluster sampling was conducted, with each cluster comprising eight households. The choice of cluster size was based on the daily performance capacity of the data collection group. The statistical framework was based on the household lists available from every health department in the provinces. The ratio of sample size to the total number of households was taken as 1:1000 (13 478 households through 1681 clusters). In total, 35014 persons (22564 from urban areas and 12450 from rural areas) in the age group 15 years and above were studied.

\section{Measures}

The 28-item General Health Questionnaire (GHQ-28) was used as a screening tool for the detection of mental disorders. This questionnaire was developed by Goldberg \& Hillier (1979) for screening for somatic symptoms, anxiety and insomnia, social dysfunction and severe depression. A review of studies on the validation of the GHQ-28 in different countries demonstrates its high validity and reliability as a screening tool of mental disorders in the community. This questionnaire was translated into the official language of Iran (Persian), which is comprehensible to almost every Iranian, and its validity and reliability were approved in an independent study (Noorbala et al, 1999). The best cutoff point, determined using the conventional scoring method and the minimum overall misclassification rate, was 6: that is, those scoring 6 and above were designated as possible cases of mental disorder. Sensitivity, specificity and overall misclassification rate for a GHQ-28 cut-off score of 6 were $84.7 \%, 93.8 \%$ and $8.2 \%$, respectively. The reliability of the GHQ-28 was assessed on a sample of 90 participants retested 1 week after the initial referral. The estimated intraclass correlation between the test-retest scores was 0.85 (Noorbala et al, 1999). To detect psychosis, epilepsy and learning disability ('mental retardation'), a simple semi-structured clinical interview with its limited validity and reliability was used.

\section{Collection of data}

This survey was implemented as a part of the National Health Survey in Iran. In each province, specially trained general practitioners from the provincial health centres visited the selected households and completed the GHQ-28 for the age group 15 years and above. Detection of cases of psychosis, epilepsy and learning disability was based on semi-structured clinical interviews by the general practitioners, and on available medical and paramedical records. 


\section{Statistical methods}

Data relating to the survey were analysed using the Statistical Package for the Social Sciences, version 8.0 for Windows. Logistic regression modelling was used to determine the factors that affect mental disorders. Mental disorders were considered as dependent variables and gender, age, education, occupation and marital status were considered as independent variables. Using the logistic regression model, the odds ratios, their level of significance and standard deviation were calculated. Backward logistic regression $\left(P_{\mathrm{e}}=0.15\right.$ and $\left.P_{\mathrm{r}}=0.2\right)$ was also used (Hosmer \& Lemshow, 1989).

\section{RESULTS}

A fifth of the population under survey (21\%, range $20.5-21.5)$ had mental disorders. The figure for women was 1.7 times that for men $(29 \% v .15 .8 \%)$. Data regarding prevalence of mental disorders in terms of gender, place of residence, age, marital status, education and occupation are presented in Table 1. Based on the logistic regression analyses (Table 2), the following conclusions can be drawn.

(a) Women had a relative risk of mental disorders of 1.632 compared with men.

(b) The risk of mental disorders increases with age.

(c) Married people were 1.142 times more at risk of mental disorders compared with unmarried people. Divorced or widowed people were 1.751 times more at risk of mental disorders compared with unmarried people.

(d) The highest risk of mental disorders was related to unemployment (unemployed people were 1.813 times more at risk of mental disorders compared with employed people). Housewives $(\mathrm{RR}=1.361)$ and farmers $(\mathrm{RR}=1.224)$ were more at risk of mental disorders compared with employed people.

(e) With increasing educational level, the risk of mental disorders decreases.

According to the clinical interviews by general practitioners of household members, $1.4 \%$ had evident learning disability, $1.2 \%$ had epilepsy and $0.6 \%$ had psychotic disorders. It also shows that $21 \%$ of the sample experienced depressive symptoms, $20.8 \%$ anxiety symptoms, $17.9 \%$ somatic symptoms and $14.2 \%$ social dysfunction symptoms.
Table I Prevalence of mental disorders in terms of demographic variables $(n=35014)$

\begin{tabular}{|c|c|c|c|}
\hline Variable & Sample size (n) & Suspected cases ( $n$ ) & Prevalence rate (\%) \\
\hline \multicolumn{4}{|l|}{ Gender } \\
\hline Male & 15506 & 2304 & 14.9 \\
\hline Female & 19508 & 5054 & 25.9 \\
\hline \multicolumn{4}{|l|}{ Place of residence } \\
\hline Rural & 12450 & 2647 & 21.3 \\
\hline Urban & 22564 & 4711 & 20.9 \\
\hline \multicolumn{4}{|l|}{ Age group (years) } \\
\hline $15-24$ & II 448 & 2017 & 17.6 \\
\hline $25-44$ & 14367 & 2843 & 19.8 \\
\hline $45-64$ & 6136 & 1534 & 25.0 \\
\hline $65+$ & 3063 & 964 & 31.5 \\
\hline \multicolumn{4}{|l|}{ Marital status } \\
\hline Unmarried & 9899 & 1783 & 18.0 \\
\hline Married & 23188 & 4757 & 20.5 \\
\hline Divorced or widowed & 1927 & 818 & 42.4 \\
\hline \multicolumn{4}{|l|}{ Occupation } \\
\hline Student & 4492 & 700 & 15.6 \\
\hline Unemployed & 4393 & 1122 & 25.5 \\
\hline Housewife & 14218 & 3839 & 27.0 \\
\hline Employed & 2778 & 354 & 12.7 \\
\hline Private sector & 6430 & 950 & 14.8 \\
\hline Farmer & 2703 & 393 & 14.5 \\
\hline
\end{tabular}

Table 2 Estimated logistic regression coefficients and odds ratios

\begin{tabular}{|c|c|c|c|c|c|}
\hline Variable & B & s.e. & $P$ & OR & $95 \% \mathrm{Cl}$ \\
\hline \multicolumn{6}{|l|}{ Marital status } \\
\hline Unmarried & - & - & - & - & - \\
\hline Married & 0.133 & 0.047 & 0.004 & 1.142 & $1.043-1.251$ \\
\hline Widowed or divorced & 0.56 & 0.053 & $<0.001$ & $\mathrm{I} .75 \mathrm{I}$ & $1.576-1.944$ \\
\hline Age & 0.01 & $0.00 \mathrm{I}$ & $<0.00 \mathrm{I}$ & 1.01 & $1.008-1.012$ \\
\hline \multicolumn{6}{|l|}{ Gender } \\
\hline Male & - & - & - & - & - \\
\hline Female & 0.49 & 0.046 & $<0.00 \mathrm{I}$ & 1.632 & I.49I-I.787 \\
\hline Education & -0.025 & 0.004 & $<0.00 \mathrm{I}$ & 0.976 & $0.968-0.983$ \\
\hline \multicolumn{6}{|l|}{ Place of residence } \\
\hline Urban & - & - & - & - & - \\
\hline Rural & 0.058 & 0.03 & 0.058 & 1.059 & $0.998-I .124$ \\
\hline \multicolumn{6}{|l|}{ Occupation } \\
\hline Employed & - & - & - & - & - \\
\hline Unemployed & 0.595 & 0.072 & $<0.001$ & 1.813 & I.575-2.085 \\
\hline Housewife & 0.309 & 0.073 & $<0.001$ & $1.36 \mathrm{I}$ & I.179-1.572 \\
\hline Student & 0.142 & 0.08 & 0.076 & 1.152 & $0.985-1.348$ \\
\hline Private & 0.026 & 0.072 & 0.717 & 1.026 & $0.892-I .182$ \\
\hline Farmer & 0.202 & 0.082 & 0.013 & 1.224 & I.043-I.437 \\
\hline Constant & -2.199 & 0.093 & $<0.001$ & & \\
\hline
\end{tabular}




\section{DISCUSSION}

\section{Prevalence}

Epidemiological surveys of mental disorders in Iran report rates varying between $11.9 \%$ and $23.8 \%$ (Noorbala et al, 2001). Comparison of prevalence rates in our survey with those detected in other surveys using different methods, tools or classifications, shows that the results of surveys using the GHQ-28 are approximately compatible. Differences in methods and tools for screening and diagnosis as well as different classification systems used and age groups studied may account for the minor differences in results.

Comparing the results with Western studies, the prevalence rate obtained in this survey is higher than rates in surveys conducted by Hoeper et al (1979), Hodiamont et al (1987) and Fones et al (1998), but lower than those obtained by Kessler et al (1994) and Lee et al (1990). It is approximately similar to prevalence rates found by Stansfeld \& Marmot (1992) and Roca et al (1999). The findings further show that the maximum rates of positive responses to questions on the GHQ-28 were for headache, distress, insomnia, sorrow and disappointment, confirming findings of other studies conducted in Iran. Anxiety and depressive symptoms were common, which is comparable with results of similar surveys in Iran and in other studies reported by Kaplan \& Sadock (2000).

\section{Gender distribution}

The study found higher prevalence rates of mental disorder in women than in men $(25.9 \%$ v. $14.9 \%)$, compatible with results of other surveys in Iran and those conducted in other countries. Gender and marital roles can be considered as possible explanations for the higher rates. The majority of women are bound to their social roles as housewives; even when women work outside the home, they still have the burden of housework. Hence, the latter group should be more subject to strains and stress; however, our research showed that working only within the home has a more serious impact on psychiatric morbidity. The fact that women in Iran are more at risk of mental disorders than is the case in Western cultures may be due to the robust effect of biological factors or to social inconveniences experienced more by women than by men. However,

\section{CLINICAL IMPLICATIONS}

The prevalence of mental disorders in Iran is comparable with most other countries.

- Mental disorders are more prevalent in women than in men; unemployment and being divorced or widowed are other main correlates of morbidity.

Iranian mental health facilities should be extended to provide for the estimated 10-12 million persons suffering from psychiatric illness.

\section{LIMITATIONS}

- This survey was conducted using the 28 -item General Health Questionnaire, which is not a structured clinical interview, and was unable to assess the prevalence of different disorders.

- Frequency of psychosis, epilepsy and learning disability was measured by general physicians using semistructured clinical interviews with limited validity and reliability.

- The sample does not include children and adolescents, who constitute a major part of the Iranian population.

A. A. NOORBALA, MD, Tehran University of Medical Sciences; S. A. BAGHERI YAZDI, MSc, Mental Health Unit, Ministry of Health and Medical Education, Tehran; M. T. YASAMY, MD, Shahid Beheshti University of Medical Sciences; K. MOHAMMAD, PhD, Epidemiology and Biostatistics Department, School of Public Health, Tehran University of Medical Sciences, Tehran, Iran

Correspondence: Dr A. A. Noorbala, Roozbeh Psychiatric Hospital, Tehran University of Medical Sciences, South Kargar Avenue, Tehran, Iran. E-mail: noorbala@irrcs.org

(First received 23 April 2003, final revision I5 July 2003, accepted 31 July 2003)

the type of such social problems may differ between cultures. The study demonstrates higher rates in rural populations than in city dwellers $(21.3 \% v .20 .9 \%)$, but the difference is not statistically significant.

\section{Age distribution}

The study revealed a significant correlation between age and the occurrence of mental disorders. Prevalence rates increase with age, supporting the results of Lee $e t$ al (1990) and Hodiamont et al (1987). This may be explained by reduction in physical vigour and the greater vulnerability of older people to stress as well as mental and physical diseases. This finding is not compatible with those of similar surveys conducted in Iran showing higher rates in people aged up to 45 years compared with those aged 45 and above (Noorbala et al, 2001).

\section{Stress}

This study supports the results of earlier studies showing higher rates of mental disorders among illiterate and semi-literate groups. Sociocultural constraints in such groups posing limits to their coping styles in the face of stress may be considered as one of the main factors. Confirming the results of other epidemiological studies in Iran (Noorbala et al, 1998), our survey's findings demonstrate higher prevalence rates of mental disorder among the married: these may be due to economic and social stress factors such as financial matters, family management and child care. The study's findings of higher rates of mental disorder among housewives and unemployed men also reported by Hodiamont et al (1987), Stansfeld \& Marmot (1992), Murthy \& Burns (1992), Bahar et al (1992) and Noorbala et al (1998) may be explained as the overall outcome of 
insufficient income, the stress of unemployment, limited social relations and monotonous lifestyle. There is also a possibility that mental disorders have contributed to unemployment.

\section{Implications for health care}

Our finding that about a fifth of the population surveyed in the age group 15 years and above suffers from mental disorders suggests that 10-12 million persons in Iran require mental health care. Taking into consideration the present number of Iranian psychiatrists (735) and the present number of Iranian mental hospital beds (7850; Yasamy et al, 2001), the need to provide appropriate staff and facilities to render mental health care is more evident than ever.

\section{ACKNOWLEDGEMENTS}

This project was supported by the Deputy of Research, Ministry of Health and Medical Education as part of the National Health and Disease Survey.

Many thanks to Professor Goldberg for providing the original GHQ-28 manual and related literature. Our appreciation goes to Dr Hosein Malekafzaly, Deputy Minister of Health and Medical Education, Research Affairs; Dr Ali Akbar Sayyari, Deputy Minister of Health and Medical Education, Health Affairs; Dr Zavaran; Dr Gouya; and the late Dr
Shahmohammadi. We also thank our colleagues Dr Seyedreza Majdzadeh, Dr Seyed Mehdi Sadathashemi, Dr Masoud Karimlu, Dr Hamid Yaghoubi, Reza Mohammad Salehi and many others for their support, efforts and patience.

\section{REFERENCES}

Bahar, E., Henderson, A. S. \& Mackinnon, A. J. (1992) An epidemiological study of mental health and socioeconomic conditions in Sumatra, Indonesia. Acta Psychiatrica Scandinavica, 85, 257-263.

\section{Fones, C. S., Kua, E. H., Ko, S. M., et al (1998)}

Studying the mental health of Singapore. Singapore Medical Journal, 53, 25I-260.

Goldberg, D. \& Hillier, V. F. (1979) A scaled version of the General Health Questionnaire. Psychological Medicine, 9, |3|-145.

Hodiamont, P., Peer, N. \& Syben, N. (1987)

Epidemiological aspects of psychiatric disorder in a Dutch health area. Psychological Medicine, 17, 227-24I.

Hoeper, E. W., Nycz, G. R., Cleary, P. D., et al (1979) Estimated prevalence of RDC mental disorders in primary medical care. International Medical Journal of Mental Health, 8, 6-15.

Hosmer, D. \& Lemshow, S. (1989) Applied Logistic Regression. New York: John Wiley

Kaplan, H. \& Sadock, B. J. (2000) Comprehensive Textbook of Psychiatry (7th edn). Baltimore, MD: Lippincott, Williams \& Wilkins.

Kessler, R. C., McGonagle, K. A., Hao, S., et al (1994) Lifetime and 12 month prevalence of DSM-III-R psychiatric disorders in the United States. Results from the National Comorbidity Survey. Archives of General Psychiatry, 5I, 9-19.

Lee, C. K., Kwak, Y. S., Yamamoto, J., et al (1990) Prevalence of mental disorders in South Korea. Journal of Nervous and Mental Disease, 178, 242-246.

Murray, C. \& Lopez, A. (1996) Global Burden of Disease: A Comprehensive Assessment of Mortality and Disability from Diseases, Injuries, and Risk Factors in 1990 and Projected to 2020. Geneva: WHO.

Murthy, R. S. \& Burns, N. (1992) Community Mental Health. Bangalore: National Institute of Mental Health and Neuroscience (NIHMANS) Publications.

Noorbala, A. A., Mohammad, K. \& Bagheri Yazdi, S. A. (1998) A survey of psychiatric disorders in Tehran city. Hakim Magazine, 4, 212-223.

Noorbala, A. A., Mohammad, K., Bagheri Yazdi, S. A., et al (1999) Validation of GHQ-28 in Iran. Hakim Magazine, 5, 101-110.

Noorbala, A. A., Mohammad, K., Yasamy, M.T., et al (200I) A View of Mental Health in Iran. Tehran: Iranian Red Crescent Society Publications.

Roca, M., Gili, M., Ferrer, V., et al (1999) Mental disorders on the Island of Formentera, Spain. Social Psychiatry and Psychiatric Epidemiology, 34, 4I0-4I5.

Stansfeld, S. A. \& Marmot, M. G. (1992) Social class and minor psychiatric disorder in British civil servants: a validated screening survey using the General Health Questionnaire. Psychological Medicine, 22, 739-749.

Yasamy, M. T., Shahmohammadi, D., Bagheri Yazdi, S. A., et al (200I) Mental health in the Islamic Republic of Iran: achievements and areas of need. Eastern Mediterranean Health Journal, 7, 38I-39l. 